\title{
LTR_FINDER_parallel: parallelization of LTR_FINDER enabling rapid identification of long terminal repeat retrotransposons
}

\author{
Shujun Ou(D) and Ning Jiang ${ }^{*}$ (D)
}

\begin{abstract}
Annotation of plant genomes is still a challenging task due to the abundance of repetitive sequences, especially long terminal repeat (LTR) retrotransposons. LTR_FINDER is a widely used program for the identification of LTR retrotransposons but its application on large genomes is hindered by its single-threaded processes. Here we report an accessory program that allows parallel operation of LTR_FINDER, resulting in up to 8500X faster identification of LTR elements. It takes only 72 min to process the $14.5 \mathrm{~Gb}$ bread wheat (Triticum aestivum) genome in comparison to 1.16 years required by the original sequential version. LTR_FINDER_parallel is freely available at https://github. com/oushujun/LTR_FINDER_parallel.
\end{abstract}

Keywords: Genome annotation, Transposable element, LTR retrotransposon, LTR_FINDER

\section{Introduction}

Transposable elements (TEs) are the most prevalent components in eukaryotic genomes. Among different TE classes, long terminal repeat (LTR) retrotransposons, including endogenous retroviruses (ERVs), is one of the most repetitive TEs due to their high copy numbers and large element sizes [1]. LTR retrotransposons are found in almost all eukaryotes including plants, fungi, and animals, but are most abundant in plant genomes [2]. For example, LTR retrotransposons contribute more than 65 and $70 \%$ to the genomes of bread wheat (Triticum aestivum) and maize (Zea mays), respectively [1].

Annotation of LTR retrotransposons relies primarily on de novo approaches due to their highly diverse terminal repeats. For this purpose, many computational programs have been developed in the past two decades. LTR FINDER is one of the most popular LTR search engines [3], and the prediction quality out-performs counterpart programs [1]. However, LTR_FINDER runs on a single thread and is prohibitively slow for large genomes with long contigs, preventing its application in those species. In this study, we applied the "divide and conquer" approach to simplify and parallel the annotation task for the original

\footnotetext{
* Correspondence: jiangn@msu.edu

Department of Horticulture, Michigan State University, East Lansing, MI 48824, USA
}

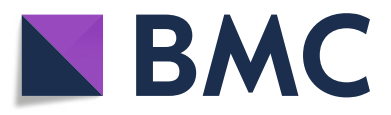

LTR_FINDER and observed an up to 8500 times speedup for analysis of known genomes.

\section{Methods}

We hypothesized that complete sequences of highly complex genomes may contain a large number of complicated nested structures that exponentially increase the search space. To break down these complicated sequence structures, we split chromosomal sequences into relatively short segments $(1 \mathrm{Mb})$ and executes LTR FINDER in parallel. We expect the time complexity of LTR_FINDER_parallel is $O(\mathrm{n})$. For highly complicated regions (i.e., centromeres), one segment could take a rather long time (i.e., hours). To avoid extended operation time in such regions, we used a timeout scheme (300 s) to control for the longest time a child process can run. If timeout, the $1 \mathrm{Mb}$ segment is further split into $50 \mathrm{~Kb}$ segments to salvage LTR candidates. After processing all segments, the regional coordinates of LTR candidates are converted back to the genome-level coordinates for the convenience of downstream analyses.

LTR_FINDER_parallel is a Perl program that can be "download and run" and does not require any form of installation. We used the original LTR_FINDER as the search engine which is binary and also installation free. Based on our previous study [1], we applied the

(c) The Author(s). 2019 Open Access This article is distributed under the terms of the Creative Commons Attribution 4.0 International License (http://creativecommons.org/licenses/by/4.0/), which permits unrestricted use, distribution, and reproduction in any medium, provided you give appropriate credit to the original author(s) and the source, provide a link to the Creative Commons license, and indicate if changes were made. The Creative Commons Public Domain Dedication waiver (http://creativecommons.org/publicdomain/zero/1.0/) applies to the data made available in this article, unless otherwise stated. 
Table 1 Benchmarking the performance of LTR_FINDER_parallel

\begin{tabular}{|c|c|c|c|c|}
\hline Genome & Arabidopsis & Rice & Maize & Wheat \\
\hline Version & TAIR10 & MSU7 & AGPv4 & CS1.0 \\
\hline Size & $119.7 \mathrm{Mb}$ & $374.5 \mathrm{Mb}$ & $2134.4 \mathrm{Mb}$ & $14,547.3 \mathrm{Mb}$ \\
\hline Original memory (1 thread ${ }^{\mathrm{a}}$ ) & 0.37 Gbyte & 0.55 Gbyte & 5.00 Gbyte & 11.88 Gbyte $^{\mathrm{b}}$ \\
\hline Parallel memory (36 threads ${ }^{\mathrm{a}}$ ) & 0.10 Gbyte & 0.12 Gbyte & 0.82 Gbyte & 17.67 Gbyte \\
\hline Original time (1 thread) & $0.58 \mathrm{~h}$ & $2.1 \mathrm{~h}$ & $448.5 \mathrm{~h}$ & $10,169.3 h^{b}$ \\
\hline Parallel time (36 threads) & $6.4 \mathrm{~min}$ & $2.6 \mathrm{~min}$ & $10.3 \mathrm{~min}$ & $71.8 \mathrm{~min}$ \\
\hline Speed up & $5.4 X$ & $48.5 x$ & $2613 x$ & $8498 x$ \\
\hline \# of LTR candidates (1 thread) & 226 & 2851 & 60,165 & 231,043 \\
\hline \# of LTR candidates (36 threads) & 226 & 2834 & 59,658 & 237,352 \\
\hline$\%$ difference in candidate \# & $0.00 \%$ & $0.60 \%$ & $0.84 \%$ & $-2.73 \%$ \\
\hline
\end{tabular}

${ }^{a}$ Intel(R) Xeon(R) CPU E5-2660 v4 @ 2.00GHz

b LTR_FINDER was run on each chromosome; the maximum memory and the total time are shown

optimized parameter for LTR_FINDER (-w 2 -C -D 15000 -d 1000 -L 7000 -1 100 -p 20 -M 0.85), which identifies long terminal repeats ranging from 100 to 7000 bp with identity $\geq 85 \%$ and interval regions from 1 to $15 \mathrm{~Kb}$. The output of LTR_FINDER_parallel is convertible to the popular LTRharvest [4] format, which is compatible to the high-accuracy post-processing filter LTR_retriever [1].

\section{Results}

To benchmark the performance of LTR_FINDER_parallel, we selected four plant genomes with sizes varying from $120 \mathrm{Mb}$ to $14.5 \mathrm{~Gb}$, which are Arabidopsis thaliana (version TAIR10) [5], Oryza sativa (rice, version MSU7) [6, 7], Zea mays (maize, version AGPv4) [8], and Triticum aestivum (wheat, version CS1.0) [9], respectively. Each of the genomes was analyzed both sequentially ( 1 thread) and in parallel (36 threads) with wall clock time and maximum memory recorded.

Using our method, we observe $5 \mathrm{X}-8500 \mathrm{X}$ increase in speed for plant genomes with varying sizes (Table 1). For the $14.5 \mathrm{~Gb}$ bread wheat genome, the original LTR FINDER took $10,169 \mathrm{~h}$, or 1.16 years, to complete, while the multithreading version completed in $72 \mathrm{~min}$ on a modern server with 36 threads, demonstrating an 8500X increase in speed (Table 1). Even we analyzed each wheat chromosome separately, the original LTR FINDER still took 20 days on average to complete. Among the genomes we tested, the parallel version of LTR_FINDER produced slightly different numbers of LTR candidates when compared to those generated using the original version $(0-2.73 \%$; Table 1$)$, which is likely due to the use of the dynamic task control approach for processing of heavily nested regions. By filtering out LTR candidates in the rice genome with LTR retriever [1], we found a difference of 28 LTR elements (out of $\sim 1950$ filtered elements) between results from the original version and the parallel version of LTR FINDER. Of these 28 elements, 25 of them were located at the sequence split sites. However, all of the 28 elements were represented by similar full-length copies identified from other locations in the genome, indicating little loss in terms of the coverage of final library for LTR retrotransposons. Given the substantial speed improvement (Table 1), we consider the parallel version to be a promising solution for large genomes.

\section{Abbreviations}

ERV: Endogenous retrovirus; LTR retrotransposon: Long terminal repeat retrotransposon; TE: Transposable element

\section{Acknowledgements}

We wish to acknowledge Matthew Hufford (lowa State University) and Candice Hirsch (University of Minnesota) for helpful feedback on a previous version of this manuscript

\section{Authors' contributions}

SO and NJ conceived this study. SO developed the code and analyzed the genomes. SO and NJ wrote and revised the manuscript. All authors read and approved the final manuscript.

\section{Funding}

This study was supported by National Science Foundation (IOS-1740874 to N.J.); United States Department of Agriculture National Institute of Food and Agriculture and AgBioResearch at Michigan State University (Hatch grant MICL02408 to N.J.).

Availability of data and materials

LTR_FINDER_parallel is freely available at https://github.com/oushujun/LTR_ FINDER parallel.

Ethics approval and consent to participate

Not applicable.

Consent for publication

Not applicable.

Competing interests

The authors declare that they have no competing interests. 
Received: 27 September 2019 Accepted: 5 December 2019

Published online: 12 December 2019

\section{References}

1. Ou S, Jiang N. LTR_retriever: a highly accurate and sensitive program for identification of long terminal repeat Retrotransposons. Plant Physiol. 2018; 176(2):1410-22.

2. Bennetzen $J$, Wang $H$. The contributions of transposable elements to the structure, function, and evolution of plant genomes. Annu Rev Plant Biol. 2014;65(1):505-30.

3. Xu Z, Wang H. LTR_FINDER: an efficient tool for the prediction of full-length LTR retrotransposons. Nucleic Acids Res. 2007;35(Web Server issue):W265-8.

4. Ellinghaus D, Kurtz S, Willhoeft U. LTRharvest, an efficient and flexible software for de novo detection of LTR retrotransposons. BMC Bioinformatics. 2008;9(1):18.

5. Arabidopsis Genome Initiative. Analysis of the genome sequence of the flowering plant Arabidopsis thaliana. Nature. 2000;408(6814):796-815.

6. International Rice Genome Sequencing Project. The map-based sequence of the rice genome. Nature. 2005;436(7052):793-800.

7. Kawahara Y, et al. Improvement of the Oryza sativa Nipponbare reference genome using next generation sequence and optical map data. Rice. 2013; 6(1):1-10.

8. Jiao Y, et al. Improved maize reference genome with single-molecule technologies. Nature. 2017;546:524-7.

9. International Wheat Genome Sequencing Consortium. Shifting the limits in wheat research and breeding using a fully annotated reference genome. Science. 2018;361(6403):eaar7191.

\section{Publisher's Note}

Springer Nature remains neutral with regard to jurisdictional claims in published maps and institutional affiliations.

Ready to submit your research? Choose BMC and benefit from:

- fast, convenient online submission

- thorough peer review by experienced researchers in your field

- rapid publication on acceptance

- support for research data, including large and complex data types

- gold Open Access which fosters wider collaboration and increased citations

- maximum visibility for your research: over $100 \mathrm{M}$ website views per year

At BMC, research is always in progress.

Learn more biomedcentral.com/submissions 